Check for updates

Cite this: RSC Adv., 2017, 7, 32866

Received 31st March 2017 Accepted 7th June 2017

DOI: 10.1039/c7ra03736h

rsc.li/rsc-advances

\section{Predictive modeling and validation of arsenite removal by a one pot synthesized bioceramic buttressed manganese doped iron oxide nanoplatform $\dagger$}

\begin{abstract}
Nitesh Dhiman, ${ }^{\text {ab }}$ Markandeya, ${ }^{\text {cd }}$ Faimy Fatima, ${ }^{a}$ Prem N. Saxsena, ${ }^{e}$ Somendu Roy, ${ }^{f}$ Prashant K. Rout ${ }^{\mathrm{g}}$ and Satyakam Patnaik (D) *ab

In the present study, a series of bioceramic capped manganese doped superparamagnetic iron oxide (SPIONs) nanoparticles (mHAP NPs) were synthesized by one pot in situ reduction. The aging of the bioceramic on the SPIONs was optimized to achieve variation in functionality, morphology, magnetic susceptibility, shape and size of the NPs and ultimately remediation effectiveness of arsenite; As(III). Results indicate that among various synthesized NPs, the $6 \mathrm{~h}$ aged mHAP NPs with 27 emu magnetic susceptibility, $57.30 \mathrm{~m}^{2} \mathrm{~g}^{-1}$ surface area and $75.64 \AA$ average pore diameter offer the best option as an adsorbent for posthaste removal of As(III) from synthetically spiked water. Further, predictive modeling using response surface based Central Composite Design (CCD) was applied to achieve and optimize process parameters for the removal of As(III) by mHAP NPs keeping variable operational parameters to a minimum in batch experiments. The individual and collective effect of four process parameters, i.e. $\mathrm{pH}$, mHAP NP dose, contact time and initial As(III) concentration on As(III) adsorption were studied. The results from statistical design signify that, with $0.2 \mathrm{~g} \mathrm{~L}^{-1}$ of above mHAP NPs adsorbent dose, $98 \%$ As(III) (initial concentration $0.1-0.4 \mathrm{mg} \mathrm{L}^{-1}$ ) removal was possible in $210 \mathrm{~min}$ at $\mathrm{pH} 6.5$ which is well within the prescribed value as per WHO guidelines. The adsorption process of As(III) onto mHAP NPs showed excellent correlation with a Langmuir isotherm with a maximum adsorption capacity of $12.0 \mathrm{mg} \mathrm{g}^{-1}$. Kinetic and thermodynamic studies reveal pseudo-second-order kinetics with an exothermic and spontaneous adsorption of As(III) on the synthesized adsorbent. Cyclic regeneration of mHAP NPs indicated positive impact in remediation technology at low production cost.
\end{abstract}

\section{Introduction}

${ }^{a}$ Water Analysis Laboratory, Nanotherapeutics \& Nanomaterial Toxicology Group, CSIR-Indian Institute of Toxicology Research (CSIR-IITR), Vishvigyan Bhawan, 31, Mahatma Gandhi Marg, Lucknow-226001, Uttar Pradesh, India. E-mail: satyakampatnaik@iitr.res.in; satyakampatnaik@yahoo.com; Tel: +918960420042 ${ }^{b}$ Academy of Scientific and Innovative Research (AcSIR), CSIR-IITR Campus, Lucknow, India

${ }^{\circ}$ Department of Civil Engineering, Institute of Engineering \& Technology, Lucknow226021, India

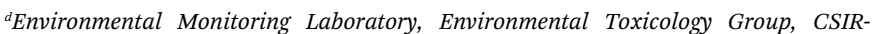
Indian Institute of Toxicology Research (CSIR-IITR), Vishvigyan Bhawan, 31, Mahatma Gandhi Marg, Lucknow-226001, Uttar Pradesh, India

${ }^{e}$ Advanced Imaging Facility, CSIR-Indian Institute of Toxicology Research (CSIR-IITR), Vishvigyan Bhawan, 31, Mahatma Gandhi Marg, Lucknow-226001, Uttar Pradesh, India

${ }^{f}$ Analytical Chemistry Division, CSIR-Indian Institute of Toxicology Research (CSIRIITR), Vishvigyan Bhawan, 31, Mahatma Gandhi Marg, Lucknow-226001, Uttar Pradesh, India

${ }^{g}$ Phytochemistry Department, CSIR-Central Institute of Medicinal and Aromatic Plants, Near Kukrail Picnic Spot, Lucknow-226015, Uttar Pradesh, India

$\dagger$ Electronic supplementary information (ESI) available. See DOI: 10.1039/c7ra03736h
Arsenic (As), the metalloid, is ubiquitous in air, soil, rocks and ground water in more than 200 mineralogical species with $60 \%$ as arsenates, $20 \%$ as sulfides and rest in the form of sulfo salts, arsenides, arsenates, oxides, silicates, and arsenic. The predominant species of As in drinking water are either arsenite $\operatorname{As}(\mathrm{III})$, or arsenate $\mathrm{As}(\mathrm{v})$, depending on redox conditions, $\mathrm{pH}$, and microbial activity and are considered by the WHO and USEPA as a first priority pollutant owing to their high toxicity. ${ }^{1,2}$ Several natural processes along with As dependent industrial applications coupled with anthropogenic activities exponentially enhance the direct or indirect distribution of As in water bodies. ${ }^{3,4}$ Through diverse pathways As exerts its toxicity including impairment of cellular respiration, uncoupling of oxidative phosphorylation, binding with sulfhydryl groups of proteins and enzymes etc. Sustained As exposure to humans leads to functional impairment of the cardiovascular system, gastrointestinal and respiratory tract, hematopoietic and nervous systems. Chronic and long term exposure to As is also 
known to induce tumors in several organs including the kidneys, liver, bladder, and skin. ${ }^{3,4}$ Cumulatively, As is categorized as a group 1 carcinogen by the International Agency for Research on Cancer (IARC, 1987) ${ }^{5}$ and consequently, the maximum contaminant level (MCL) of As in drinking water has a provision of $10 \mu \mathrm{g} \mathrm{L}^{-1}$ set on the guidelines of WHO and USEPA. ${ }^{6}$ According to USEPA, 1988 As(III) is about an order of magnitude more potent than As(v) to create teratogenic effects. ${ }^{7}$ Epidemiological studies showed metals containing drinking water is preventable cause of death and numerous diseases. Arsenic in inorganic form considered as strong carcinogens (group 1 human carcinogens) which is identified in water sources, especially in Bangladesh, India, China, USA, Myanmar, Pakistan, Mexico, Chile and Argentina. A variety of treatment processes has been developed for As exclusion such as coagulation, advanced oxidation process (AOP), ion exchange, membrane filtration, electrocoagulation, biological process and adsorption. Adsorption is economically viable, flexible, easy accessible process where prominent adsorbate interacts with surface of adsorbent by physical or chemical factor.,8-11 Recently, nanoparticles (NPs) based adsorbents are gaining much attentions against conventional remediation processes of wastewater due to their vast specific surface area when compared to respective bulk. Studies showed that metal oxides in particular iron oxides, manganese oxides, aluminum oxides, titanium oxides, magnesium oxides and cerium oxides NPs have promising potential for heavy metals removal from aqueous systems. ${ }^{\mathbf{1 2 - 1 4}}$

In particular, super paramagnetic iron oxide (SPIONs) based immobilization technology has superior prospect for clean-up process due to ease of separation and the fact that it can decontaminate a large volume of wastewater in a very short period with no secondary adulterant. ${ }^{15,16}$ However, bare magnetite NPs are highly susceptible to air oxidation, acidic leaching, have low adsorption capacity and also prone to selfaggregated in aqueous systems. To subjugate these limitations and to facilitate the adsorption affinity and specific ion separation, different functional groups and polymer coating are preferably applied onto or in form of composites such as silica, carbon based materials and polymers (both synthetic and natural) to SPIONs. ${ }^{17,18}$ Recently, Liu et al. (2008) demonstrated humic acid coated SPIONs for the removal of toxic $\mathrm{Hg}$ (II), $\mathrm{Pb}$ (II), $\mathrm{Cd}(\mathrm{II})$, and $\mathrm{Cu}(\mathrm{II})$ from water $^{19}$ Furthermore, hydroxyapatite [HAP: $\mathrm{Ca}_{10}\left(\mathrm{PO}_{4}\right)_{6}(\mathrm{OH})_{2}$ ] a principal mineral constituent of natural bones and teeth received much attention recently, due to its potential applicability in biomedical research. ${ }^{20}$ HAP owing to its crystal structure and chemical composition preferentially shows a high capacity for ion exchange with heavy metals, high stability toward oxidizing and reducing agents and have extensively used in remediation process. ${ }^{21}$ However, bulk of the studies pertaining to remediation where SPION's have been used primarily focussed on removal As(v), rather than the more toxic trivalent As(III). Recently, Shan and Tong has explored Fe-Mn binary oxide (FMBO) coated magnetic NPs for removal of $\mathrm{As}(\mathrm{III})$ through oxidation process. ${ }^{22}$ In the present study hydroxyapatite capped manganese doped SPIONs were synthesized via in situ reduction process at different ageing period. We opted for manganese doping as it enhances the magnetic susceptibility of the SPIONs. The synthesized NPs were selected on the basis of magnetic susceptibility for easy and rapid removal. Based on prefatory analysis, Central Composite Response Surface Design (CCD) originated from Response Surface Methodology (RSM) was employed to optimize process parameters such as $\mathrm{pH}$, mHAP dose, contact time and initial As(III) concentration; collectively to achieve maximum percentage removal of As(III). Moreover, equilibrium isotherm, kinetic and thermodynamic studied has been done to support the favorable and effective removal of As(III) by the synthesized and optimized mHAP NPs. To the best of our knowledge this is first of its kind pre-optimization study involving As(III) removal onto Mn doped SPIONs nanoplatform. Central Composite Response Surface Design (CCD) was applied as a tool to summarize experimental setup with variable physical parameters for ensuring water safety and provided invaluable information and feasibility of this new opportunities for remediation approaches.

\section{Methodology and design of experiment}

\subsection{Materials}

Ferric(III) chloride hexahydrate ACS reagent, 97\% (Sigma Aldrich), manganese(II) chloridetetrahydrate ACS reagent $>98 \%$, calcium nitrate tetrahydrate $\mathrm{AR}$, arsenic trioxide pract (SD Fine Chem Ltd), disodium dihydrogen phosphate dihydrate, liquor ammonia; 25\% ammonia (Fisher Scientific), were used without any purification.

All reagents and stock solution were prepared in Milli Q.

\subsection{Quality assurance}

AR grade chemicals and reagents were used in whole study for elimination of unwanted impurities. Instruments were calibrated and validated as per National Accreditation Board for testing and calibration Laboratories (NABL) norms to avoid uncertainty. Entire sets of experiments were carried out in triplicate for maintain accuracy and precision.

\subsection{Stock solution of arsenite (As(III))}

Stock As(III) solution ( $1 \mathrm{~mL}=1000 \mu \mathrm{g}$ As) was prepared according to American Public Health Association (APHA) 2012 method by dissolving $0.1320 \mathrm{~g}$ of $\mathrm{As}_{2} \mathrm{O}_{3}$ in a mixture of $50 \mathrm{~mL}$ of Milli Q water and $1 \mathrm{~mL} \mathrm{NH} \mathrm{NH}_{4}{ }^{23}$ Mixture was heated gently to dissolve the metal salt, cooled and acidified with $2 \mathrm{~mL} \mathrm{HNO}_{3}$ prior to making up the volume with $100 \mathrm{~mL}$ of Milli Q.

\subsection{Synthesis of mHAP NPs adsorbent}

In this study, motivated by the aforementioned situation, the simultaneous synthesis of Mn doped SPION's, HAP and mHAP NPs were achieved by one pot co-precipitation method in alkaline media. Briefly, $100 \mathrm{~mL}$ of Milli Q was taken in $500 \mathrm{~mL}$ RB flask and degassed for half an hour followed by successive addition of manganese(II) chloride tetrahydrate $(2 \mathrm{mmol})$ and 
ferric(III) chloride hexahydrate $(4 \mathrm{mmol})$ to $\mathrm{RB}$ with stirring at RT. After thorough mixing the reaction vessel was assembled with a reflux condenser and $25 \mathrm{~mL}$ ammonia solution was added while allowing the temperature to rise to $100{ }^{\circ} \mathrm{C}$ with stirring for an hour. Thereafter, calcium nitrate tetrahydrate (16.8 mmol) and disodium dihydrogen phosphate dihydrate (10 mmol) were added in $1.67: 1$ ratio maintaining $\mathrm{pH} 11$. The mixture was further kept at $100{ }^{\circ} \mathrm{C}$ for $2 \mathrm{~h}$ and left for ageing $(6$ and $12 \mathrm{~h}$ ). Native Mn doped SPIONs and HAP were synthesized in the same manner without adding $\mathrm{Ca}^{2+}$ and $\mathrm{PO}_{4}{ }^{3-}$ salts and magnetic NPs precursors respectively. Obtained NPs were neutralized with repetitive Milli $\mathrm{Q}$ washing using a permanent magnet and oven dried at $100{ }^{\circ} \mathrm{C}$.

\subsection{Characterizations of mHAP NPs}

The Attenuated Total Reflectance-Fourier Transform Infrared (ATR-FTIR) spectra of Mn doped SPIONs, HAP and hydroxyapatite capped SPIONs (mHAP) were recorded using Thermo Fischer (Nicolet, iS5, USA) equipped with diamond ATR crystal. The spectra were recorded in $4000-350 \mathrm{~cm}^{-1}$ spectral range at a rate of 16 scans per min. Transmission Electron Microscope (TEM) (FEI Technai G-2 spirit, Netherland) operated at $80 \mathrm{kV}$ with $15000 \times$ magnifications to determine NPs size. The surface morphology and qualitative elemental analysis of Mn doped SPIONs and mHAP NPs before and after treating with As(III) were investigated under Scanning Electron Microscope (SEM) and Energy Dispersive X-ray (EDX) point analysis using field emission scanning electron microscope coupled with Energy Dispersive X-ray (SEM, Quanta FEG 450, FEI, Netherland) by mounting the samples on metallic stub using double side tape, platinum coating was applied with sputter coated (SC $7620 \mathrm{mini}$ sputter coated Quaram Technology, Ltd, UK) on the sample to make conductive for better resolution. Room temperature magnetization measurements of $\mathrm{Mn}$ doped $\mathrm{Fe}_{2} \mathrm{O}_{3}, 6 \mathrm{~h}$ aged mHAP NPs and $12 \mathrm{~h}$ aged mHAP NPs were carried out Vibrating Sample Magnetometer (VSM, ADE magnetic, USA). The size distribution curves, surface potential, stability, and ion exchange mechanism of $6 \mathrm{~h}$ aged mHAP NPs were determined with Dynamic Light Scattering (DLS) approach using Zetasizer Nano-ZSP Malvern, UK equipped with HeNe laser (633 nm). Thermal stability of mHAP NPs has determined using TGA Analyzer (Mettler Toledo Star ${ }^{\mathrm{e}}$, Columbus) operating at a heating rate of $10{ }^{\circ} \mathrm{C} \min ^{-1}$ from 50 to $900{ }^{\circ} \mathrm{C}$, under steady flow of $\mathrm{N}_{2}$ gas. X-Ray Diffraction (XRD) measurement was carried out by Rigaku X-ray diffractometer (ULTIMA IV, Rigaku, Japan) with CuK X-ray source $(=1.54056 \AA)$ at a generator voltage $40 \mathrm{kV}$, a generator current $40 \mathrm{~mA}$ with the scanning rate $2^{\circ} \mathrm{min}^{-1}$. BET surface analysis, pore volume and average pore diameter of optimum magnetic susceptibility having $6 \mathrm{~h}$ aged mHAP NPs were determined by Quantachrome Instruments, USA. Before operation, the samples were degassed at $110{ }^{\circ} \mathrm{C}$ for $3 \mathrm{~h}$ under $P /$ $P_{0}=0.99402$ to remove any contaminants that may be present at the surface.

As(III) concentration measurements were done using Atomic Florescence Spectroscopy (AF-420, PG-instruments, UK). The stock standard As was prepared by using $100 \mu \mathrm{L}$ As solution
(Merck NIST traceable standard) with the addition of $1 \mathrm{~mL}$ ascorbic acid, KI solution and $1 \mathrm{~mL}$ concentrated $\mathrm{HCl}$. Obtained solution was kept remain stand in dark for $45 \mathrm{~min}$ and finally $100 \mathrm{~mL}$ Milli Q was added to make 1 ppm solution. The desired solution was prepared by serial dilution.

\subsection{Experimental procedure}

2.6.1. Adsorption experiments. To study the effect of aging time as well as to elucidate the synthetic mechanism of adsorption process, random known amount of $\mathrm{Mn}$ doped SPION's, HAP, and different aged mHAP NPs $\left(0.2 \mathrm{~g} \mathrm{~L}^{-1}\right.$ each) were applied against $\left(0.5 \mathrm{mg} \mathrm{\textrm {L } ^ { - 1 }}\right) \mathrm{As}(\mathrm{III})$ spiked water for the $180 \mathrm{~min}$ in trail batches. Furthermore, batch experiments were executed with selected mHAP NPs having maximal removal capacity and magnetic susceptibility to interpret the effects of contact time, $\mathrm{pH}$, adsorbent ( $6 \mathrm{~h}$ aged mHAP NPs) dose and adsorbate $[\mathrm{As}(\mathrm{III})]$ concentration on removal efficiency. For this, $0.010 \mathrm{~L}$ of $\mathrm{As}$ (III) solution (concentration varying from 0.2 to $4.0 \mathrm{mg} \mathrm{L}^{-1}$ ) was taken in $25 \mathrm{~mL}$ conical flask with addition of mHAP NPs ( 0.05 to $0.5 \mathrm{~g})$. The $\mathrm{pH}$ of solution was adjusted by adding $0.1 \mathrm{~N} \mathrm{NaOH}$ and $0.1 \mathrm{~N} \mathrm{H}_{2} \mathrm{SO}_{4}$ as per requirements. The contact time was varied from 5 to $300 \mathrm{~min}$ for maximum removal of As(III) from spiked water. After appropriate time interval, solution was applied in magnetic field to obtain remediate water. The percent removal of the As(III) was calculated using the following eqn (1),

$$
\text { Percent removal }=\frac{\left(C_{0}-C_{t}\right) \times 100}{C_{0}}
$$

where $C_{0}$ is the initial $\mathrm{As}(\mathrm{III})\left(\mathrm{mg} \mathrm{L}^{-1}\right)$ and $C_{t}$ is the $\mathrm{As}$ (III) concentration $\left(\mathrm{mg} \mathrm{L}^{-1}\right)$ after time $t(\mathrm{~min})$.

2.6.2. Statistical optimization. Response Surface Methodology (RSM) is factual mathematical modelization technique, used to evaluate the relationship between the controlled experimental variables and outcomes. Statistical software Design-Expert 8.0.7.1 (Stat-Ease Inc., Minneapolis, MN, USA) was employed to design the experimental parameters for maximum removal of As(III) in detail to assess the outcome of the process factors. The detailed optimization methodology is summarized in ESI (S1) $\dagger$

\section{Results and discussion}

\subsection{Characterization of synthesized mHAP NPs adsorbent}

Fig. 1(A) shows ATR-FTIR spectra of Mn doped SPIONs, HAP and different aged mHAP NPs. There are several clear cut differences in the adsorption spectra of the NPs before and after the ageing process. The commonly exhibited distinct vibration mode of $\mathrm{PO}_{4}{ }^{3-}$ group at $1095\left(\mathrm{P}-\mathrm{O} \nu_{\text {str. }}\right), 1027$ and $870 \mathrm{~cm}^{-1}(\mathrm{P}-$ O symmetric $\nu_{\text {str. }}$. A broad absorption peaks at $1427 \mathrm{~cm}^{-1}$ assigned for $\mathrm{C}-\mathrm{OH}$ deformation vibration with contribution of $\mathrm{O}-\mathrm{C}-\mathrm{O}$ symmetric stretching vibration of $\mathrm{CO}_{3}{ }^{2-}$ groups can be observed in the spectra of HAP and mHAP NPs while the same was absent in native Mn doped SPIONs. Further, all three spectra possess the characteristic Fe-O stretching at 572 and $680 \mathrm{~cm}^{-1}$ which corroborated successful synthesis of SPIONs 


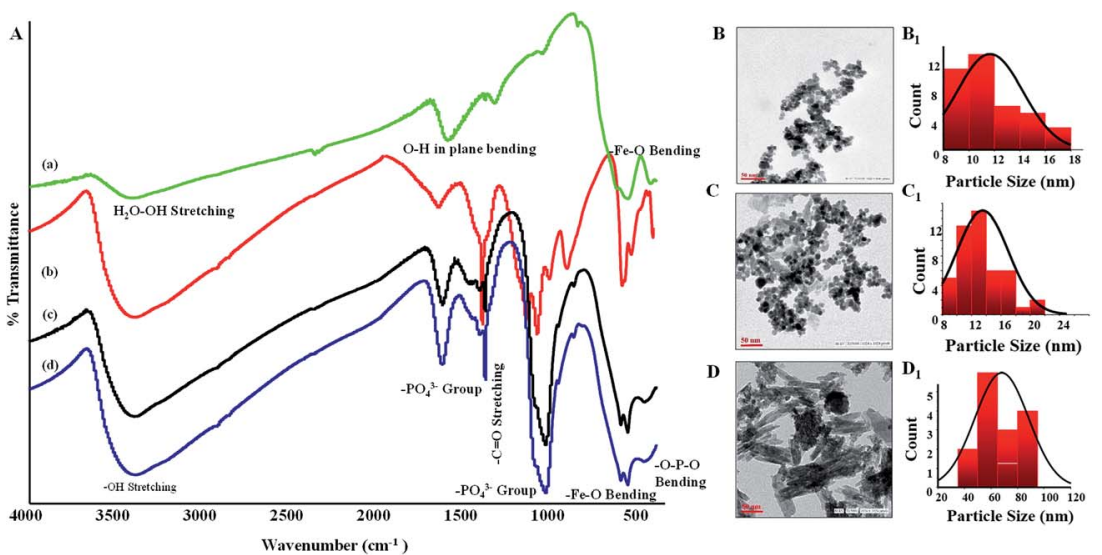

Fig. 1 (A) ATR-FTIR spectra of Mn doped SPIONs (a), HAP (b), $6 \mathrm{~h}$ aged mHAP (c), and $12 \mathrm{~h}$ aged mHAP NPs (d) and (B, B 1 ), (C, $\left.C_{1}\right)$, and (D, $\left.\mathrm{D}_{1}\right)$ are representative TEM images of Mn doped SPIONs, $6 \mathrm{~h}$ aged mHAP NPs and $12 \mathrm{~h}$ aged mHAP and their histogram respectively.

and mHAP. ${ }^{24}$ During the initial process of aging, it was found that the nucleation of HAP predominantly occurred and was almost completed until the Ca content is completely consumed by the P source.

HAP ageing was also responsible for shape and size regulation of SPIONs as illustrated by TEM images in Fig. 1(B-D). It was noticeable from Fig. $1\left(\mathrm{~B}\right.$ and $\left.\mathrm{B}_{1}\right)$ that initially synthesized Mn doped SPIONs are spherical in morphology having size around $\sim 12 \mathrm{~nm}$ which after $6 \mathrm{~h}$ ageing process expanded to $\sim 22 \mathrm{~nm}$ (Fig. 1(C and $\left.\mathrm{C}_{1}\right)$ ). Subsequently, when the SPIONs are exposed to a longer $12 \mathrm{~h}$ ageing process, the spherical shaped Mn doped SPIONs changed to rod shape with 40 to $90 \mathrm{~nm}$ size range which is shown in Fig. $1\left(\mathrm{D}\right.$ and $\left.\mathrm{D}_{1}\right)$.

The surface topography of (a) Mn doped SPIONs, (b) mHAP NPs ( $6 \mathrm{~h}$ aged) and (c) mHAP NPs (12 h aged) were effectively depicted in Fig. 2(A). The SEM micrographs shown in Fig. 2(A) (b and c; upper panel) clearly depict as ageing period is prolonged from $6 \mathrm{~h}$ to $12 \mathrm{~h}$ the roughness, porosity, flocculants surface of the mHAP concomitantly increases offering large surface, which we presume will facilitate immobilization of metals onto the adsorbent. The assumption that the metal will be adsorbed in a better way to the exfoliated mHAP NPs is corroborated in Fig. 2(A) (e and f; lower panel). The SEM micrographs showed a clear revamped smooth morphological surface of mHAP NPs after chelation with metal ions.
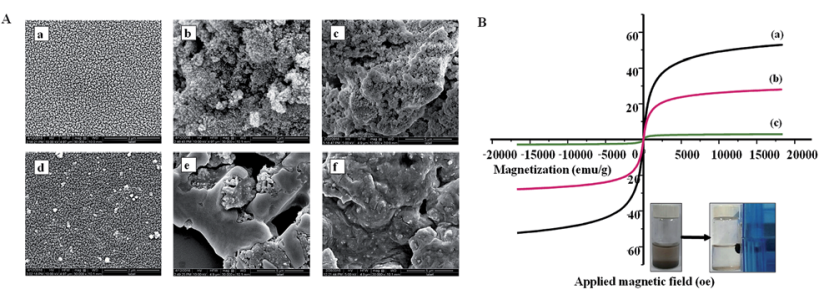

Fig. 2 (A) Representative SEM images of (a) native Mn doped SPIONs, (b) $6 \mathrm{~h}$ aged mHAP NPs and (c) $12 \mathrm{~h}$ aged mHAP NPs before As(II) adsorption; upper panel (d) native Mn doped SPIONs NPs, (e) $6 \mathrm{~h}$ aged mHAP NPs, and (f) $12 \mathrm{~h}$ aged mHAP NPs, after As(III) adsorption; lower panel: (B) VSM plots of (a) native Mn doped SPIONs (b) $6 \mathrm{~h}$ aged mHAP NPs and (c) $12 \mathrm{~h}$ aged mHAP NPs.
Towards fulfilling the aim to a more effective and rapid separation with the synthesized NPs, magnetic properties is studied using VSM and is elucidated in Fig. 2(B). All the synthesized NPs exhibited typical superparamagnetic behavior with no hysteresis, remanence and coercivity. The maximum saturation magnetization $\left(M_{\mathrm{s}}\right)$ of Mn doped SPIONs showed decline with successive ageing as shown in Fig. $2 \mathrm{~B}(\mathrm{a}-\mathrm{c})$. The magnetic susceptibility of native Mn doped SPIONs, $6 \mathrm{~h}$ aged mHAP NPs and $12 \mathrm{~h}$ aged mHAP NPs were found 52.78, 27 and $3.06 \mathrm{emu}^{-1}$, respectively. In order to reduce the synthesis steps we have added HAP precursors to the SPIONs medium in a one-pot reaction. We envisioned after certain time interval the dominant crystal growth of HAP will prohibits nucleation of Mn doped SPIONs albeit retaining sufficient magnetization. Our presumption is corroborated with the results wherein $6 \mathrm{~h}$ aged mHAP NPs shown to have 27 emu magnetic susceptibility as compared to $12 \mathrm{~h}$ aged mHAP NPs (3.06 emu). Thus the $6 \mathrm{~h}$ aged mHAP was chosen for our subsequent removal studies. The saturation magnetization values for the aged mHAP NPs were significantly lower than native SPIONs, due to substantial mass decrease of the magnetite species after functionalization. ${ }^{25}$ Various prior arts have already established the posthaste and efficient removal of As(III) by using different synthesized adsorbent. However, in order to have a better comparison and to evaluate the superiority of our developed nanosorbents, a comprehensive percentage removal of As(III) has been tabulated in Table 1. It was observed that Mn doped SPION's has less As(III) removal efficiency ( $\sim 41 \%$ ) which could be attributed to smaller adsorption capacity and lower bonding affinity. The increase in percentage removal of As(III) is evidenced from the additional modification by HAP which offers abundant binding sites for the adsorbents. However, no significant change in percentage removal was observed between $6 \mathrm{~h}(\sim 57.5 \%)$ and $12 \mathrm{~h}(\sim 59 \%)$ aged mHAP NPs while there is a decrease in magnetization.

The phase identification of synthesized $6 \mathrm{~h}$ mHAP NPs has been demonstrated with XRD analysis as shown in Fig. 3(A). The pattern showed sharp peaks with definite $2 \Theta$ value which emphasized crystalline behavior of adsorbate. Obtained pattern 
Table 1 A comparison with different synthesized adsorbent on percentage removal of As(III)

\begin{tabular}{lcc}
\hline Adsorbent & $\begin{array}{l}\text { Percentage } \\
\text { removal }\end{array}$ & $\begin{array}{c}\text { Magnetism } \\
\left(\mathrm{emu} \mathrm{g}^{-1}\right)\end{array}$ \\
\hline HAP & 36.02 & 0.0 \\
Mn doped SPIONs & 41.09 & 52.78 \\
mHAP NPs (6 h aged) & 57.57 & 27.0 \\
mHAP NPs (12 h aged) & 58.98 & 3.06 \\
\hline
\end{tabular}
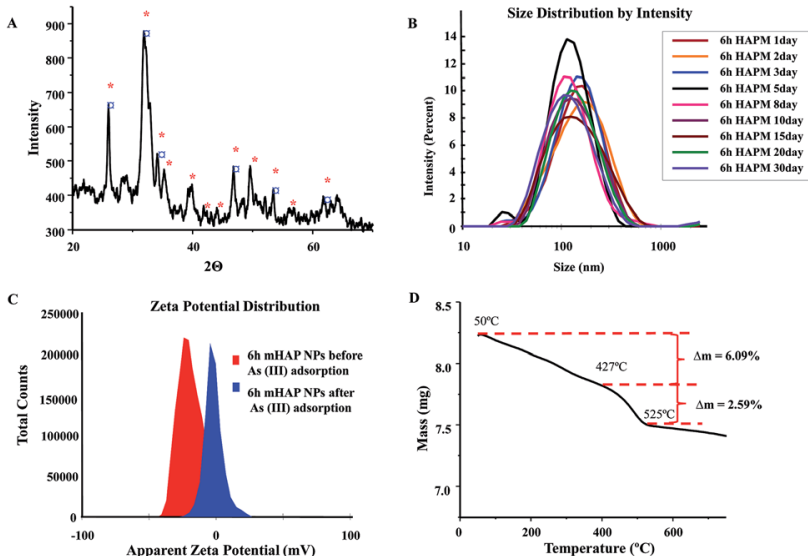

Fig. 3 (A) XRD plot of $6 \mathrm{~h}$ mHAP NPs ( $\%$ and $a$ showed HAP and Mn doped SPIONs crystalline phase respectively), (B) 30 days stability study of $6 \mathrm{~h}$ aged mHAP NPs with DLS, (C) zeta potential of mHAP NPs before and after As(III) adsorption, (D) TGA plot of $6 \mathrm{~h}$ mHAP NPs.

is well comparable with standard magnetite phase of magnetic NPs. The real world applications of a nanotechnology depends on the NPs stability vis-a-viz its degradation, confirmation and orientation changes in chemical and physical environment. The stability study of our mHAP NPs also emphasized the consistency of NPs over a time period of 30 days as shown in Fig. 3(B). Additionally, Fig. 3(C) confirms that the as optimized $6 \mathrm{~h}$ aged mHAP NPs bear high surface zeta potential $(\zeta)$ of $-21 \mathrm{mV}$ to resist and repel the NPs aggregation thereby effectively enhancing the colloidal stability in aqueous solution. It was also found that after adsorption of As(III) onto the mHAP NPs there is a significant zeta potential increment $(-1.75 \mathrm{mV}$; Fig. 3(C)), as the divalent $\mathrm{Ca}^{2+}$ ion interchange with trivalent $\mathrm{As}^{3+}$ ions supportive towards characteristic ion exchange mechanism of HAP. Thermal stability by TGA as shown in Fig. 3(D), unveiled that mHAP has high mass stability with minimal mass loss $(\Delta m$ $8.68 \%)$ till $900{ }^{\circ} \mathrm{C}$.

EDX spectra of optimized $6 \mathrm{~h}$ aged mHAP NPs before and after As(III) adsorption is shown in Fig. 4(A) and (B) respectively. The atomic percentage of $\mathrm{Ca}$ and $\mathrm{P}$ is 20.38 and 14.48 which further confirms the prescribed ratio 1.67 of $\mathrm{Ca} / \mathrm{P}$ as per basic composition of HAP. Besides, Mn and Fe atomic percentage ( 0.95 and 2.05) were also found to be in accordance to mole percentage of the reaction mixture. The chemical composition of mHAP before and after adsorption with As(III) showed lowering of the $\mathrm{Ca}^{2+}$ peak intensity of mHAP adsorbents indicating certain degree of interchange of $\mathrm{Ca}^{2+}$ with $\mathrm{As}^{3+}$ ions as reflected in the Fig. $4(\mathrm{~B}){ }^{26}$

BET specific surface area, total pore volume and average pore diameter of $6 \mathrm{~h}$ aged mHAP NPs was found to be $57.30 \mathrm{~m}^{2} \mathrm{~g}^{-1}$, $1.084 \times 10^{-1} \mathrm{~cm}^{3} \mathrm{~g}^{-1}, 75.64 \AA$, respectively. The high surface area with nanotized size provided significant affinity for metal chelation and justified the use of HAP in our final nanoformulation.

\subsection{Statistical optimization by RSM}

Detailed statistical analysis employing various models and equations are explained in ESI Section S2. $\dagger$ In a single batch system, the impacts of contact time between adsorbents and adsorbent with variable $\mathrm{pH}$, NPs dose and As(III) concentration on percentage removal of As(III) was evaluated using CCD. Total 30 runs were required in triplicate for the $\mathrm{CCD}$ and the observed percentage removal of As(III) varied between $38 \%$ and $98 \%$. The experimental parameters and their levels are summarized in Table 2.

To investigate the fitting as well as to see the better applicability of our chosen statistical model, a comparison between the actual and predicted values of As(III) removal with synthesized $6 \mathrm{~h}$ mHAP NPs was carried out and the results obtained were shown in Fig. 5(A). It was observed that there is indeed a good correlation between actual and predicated values of percentage removal. Some scattered data has been found in linear manner close to central line provided the goodness of fit and accuracy of the chosen model. Fig. 5(B) displayed the
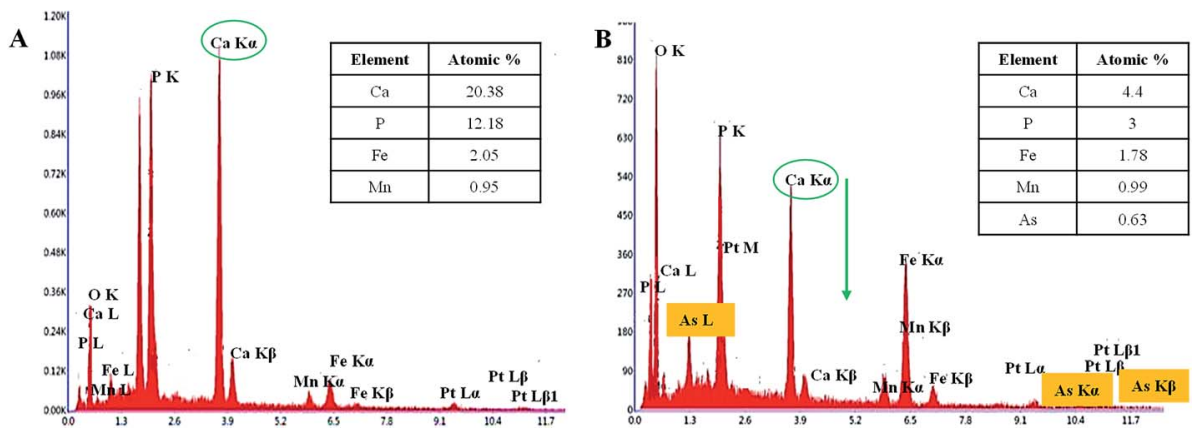

Fig. 4 Representative EDX images of $6 \mathrm{~h}$ aged mHAP NPs before (A) and after (B) adsorption of As(III) onto mHAP NPs. 
Table 2 Process variables and experimental responses for percentage removal of As(III)

\begin{tabular}{|c|c|c|c|c|}
\hline \multirow[b]{2}{*}{ Independent variables } & \multicolumn{4}{|c|}{ Levels } \\
\hline & Low $(-1)$ & Medium (0) & High (+1) & SD \\
\hline Contact time (min) (A) & 30 & 210.00 & 390 & 80.50 \\
\hline $\mathrm{pH}(\mathrm{B})$ & 2.0 & 6.00 & 10.00 & 1.79 \\
\hline $\begin{array}{l}6 \mathrm{~h} \text { aged mHAP NPs } \\
\text { dose } g \mathrm{~L}^{-1}(\mathrm{C})\end{array}$ & 0.10 & 0.20 & 0.50 & 5.14 \\
\hline As(III) conc. $\mathrm{mg} \mathrm{L}^{-1}$ (D) & 0.30 & 0.50 & 1.30 & 0.36 \\
\hline
\end{tabular}

normal percentage probability versus internally studentized residuals plots for removal of As(III) explaining the normal probability following straight line. Perturbation plot between coded units of variable factors versus percentage removal of As(III) concentration showed the comparative effects of all independent variables. Sharp curvature in contact time (A), $6 \mathrm{~h}$ mHAP NPs dose (C) and initial As(III) concentration (D) favoring measurable influence on percentage removal of As(III) concentration as compared to $\mathrm{pH}$ (B). The depiction also emphasized the order of variable on effective percentage removal of As(III) as C $>$ A $>$ D $>$ B.

\subsection{3-D response surface plots}

The mutual effects of operation parameter on outcome have been further explained by using 3-D wireframe response plots. 3-D contour plots exhibited relationship in two variables onto respective two dimensions, $x$ - and $y$-interactive response deliberated by contours. In minimax patterned response plots, from stationary point increasing either variable while decreasing other demonstrated significant feedback.

3.3.1. Optimization of $\mathbf{p H}$ and ionic strength with contact time. Adsorbate $\mathrm{pH}$ greatly impart influence on solid/liquid adsorption processes by effecting degree of ionization of the adsorbate and surface charge of the adsorbent. The synthesized
mHAP NPs possess $(\zeta)-21 \mathrm{mV}$ surface charges as demonstrated earlier having high affinity to chelate positive metal ions from solution. As(III) removal was ascertained with varied $\mathrm{pH}$ range from $\mathrm{pH} 2.0$ to 10.0 as shown in Fig. 6(A). At pre-specified As(III) concentration $\left(0.50 \mathrm{mg} \mathrm{L}^{-1}\right)$ and $6 \mathrm{~h}$ mHAP NPs dose $(0.20 \mathrm{~g}$ $\mathrm{L}^{-1}$ ) the removal efficiency of As(III) decreases with increase in $\mathrm{pH}$ which might be due to the fact that acidic $\mathrm{pH}$ promotes better electrostatic interaction between ions and surface. Subsequently, to evaluate the influence of ionic strength on adsorption of $\mathrm{As}(\mathrm{III})$, a series of experiments were carried out with $\mathrm{NaCl}$ solution (0.001-0.5 M) at pH 6.5. It was observed that the adsorption of As(III) has insignificant effect with varied ionic concentration which is also observed by Goldberg and Johnston (2001) because of inner-sphere surface complexes formation. ${ }^{27}$

3.3.2. Optimization of NPs dose (adsorbent) with contact time. Fig. 6(B) showed the interactive response of contact time and optimized mHAP NPs dose on percentage removal of As(III). A lower initial concentration of As(III) required less equilibrium adsorption time to get adsorbed onto mHAP NPs surface which was in accordance with the reported study. ${ }^{28}$ At actual factor $\mathrm{pH}$ 6 and $0.50 \mathrm{mg} \mathrm{L}^{-1}$ concentration of $\mathrm{As}(\mathrm{III})$ with increasing treatment time and NPs dose, the removal percentage reached maximum in a synergetic manner as evident from Fig. 6(B). The point related to treatment time $300 \mathrm{~min}$ with $0.35 \mathrm{~g} \mathrm{~L}^{-1}$ NPs dose displayed $100 \%$ removal whereas, 165 min contact time with $0.12 \mathrm{~g} \mathrm{~L}^{-1}$ NPs dose was effective only for around $65 \%$ removal. As a logical outcome the above results substantiate the fact that the adsorbate could get attached onto vacant adsorbent surface with the passage of time until saturation. To attempt maximum removal of absorbates it is necessary to have sufficient free binding surfaces within a timeframe of application.

3.3.3. Optimization of initial As(III) concentration with contact time. The 3-D contour plot shown in Fig. 6(C) represent the effect of initial As(III) concentration with contact time at $0.20 \mathrm{~g} \mathrm{~L}^{-1}$ NPs dose and $\mathrm{pH} 6$ on percentage removal of As(III). Fig. 6(C) showed that, at the end of 120 min near about $75 \%$ removal has been achieved which gets a further boost up to
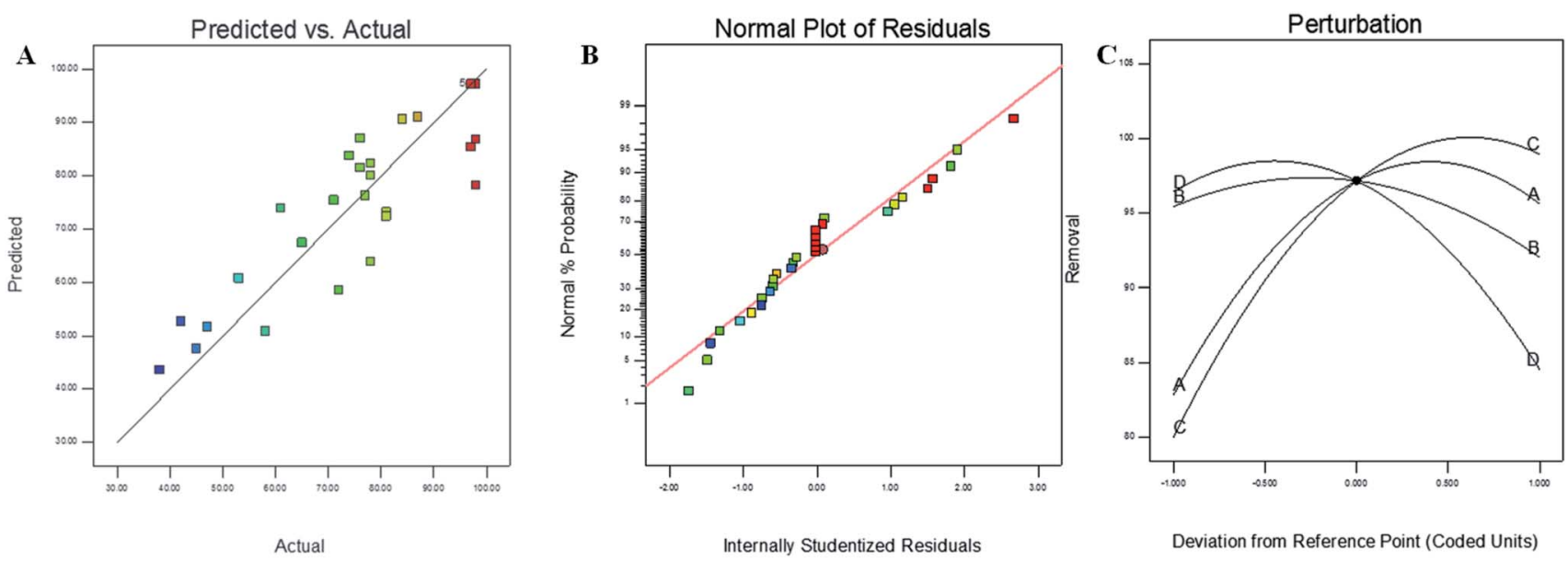

Fig. 5 Design expert plot (A) plot of the actual vs. predicted values of the percentage removal of As(III) concentration, (B) plot of the probability vs. studentized residual values of the percentage removal of As(III) concentration and (C) plot of the perturbation and percentage removal of As(III) concentration. 

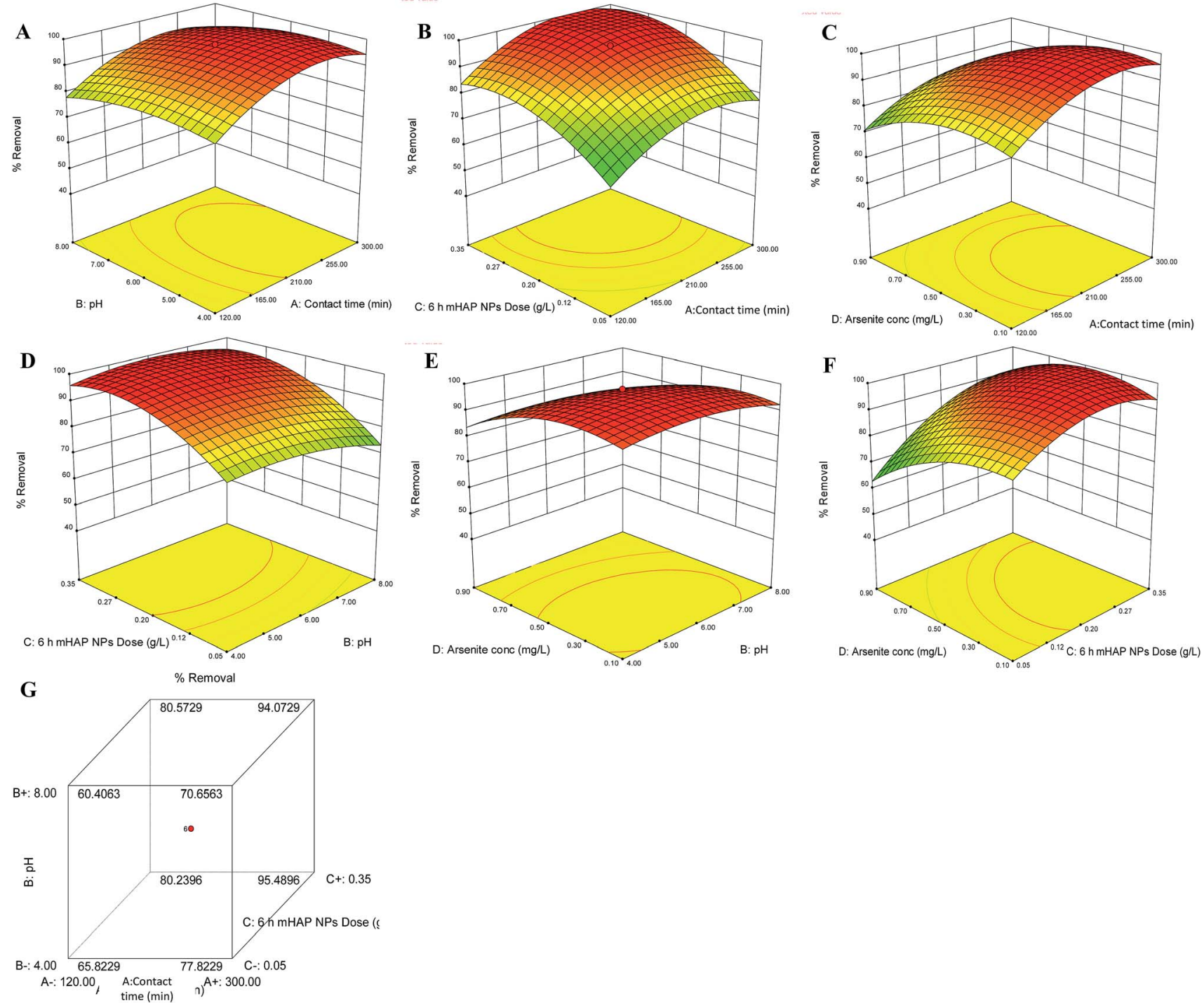

Fig. 6 3-D response surface plots of the effects of (A) pH, (B) mHAP NPs dose, and (C) initial As(III) concentration with contact time, (D) mHAP NPs dose, and (E) As(III) concentration with $\mathrm{pH}(\mathrm{F})$ initial As(III) concentration with mHAP NPs dose and (G) cumulative effect of all variable parameters.

reach a maxima beyond $165 \mathrm{~min}$. The observed phenomenon might be arising due to certain colloison occuring between absorbate to adsorbate and adsorbate to adsorbent during the initial phase of adsorption which hindered attainment of the equilibrium.

3.3.4. Optimization of NPs dose with pH. Fig. 6(D) explained that at actual factor: contact time $210 \mathrm{~min}$ and $0.50 \mathrm{mg} \mathrm{\textrm {L } ^ { - 1 }} \mathrm{As}(\mathrm{III})$, an inverse corelation was observed i.e., higher NPs dose with low $\mathrm{pH}$ condition augment the removal effeciency.

3.3.5. Optimization of As(III) concentration with pH. At the actual factor of variables such as $210 \mathrm{~min}$ and $0.20 \mathrm{~g} \mathrm{~L}^{-1}$ dose the response on removal has been verified by another two variables; As(III) concentration and pH. As shown in Fig. 6(E), it was observed at fixed As(III) concentration with low $\mathrm{pH}$ higher removal is favored compared to high $\mathrm{pH}$. All the response is result of regression equation.
3.3.6. Optimization of initial As(II) concentration with NPs dose. It was evident from Fig. 6(F) that 3-D response plots of initial As(III) concentration and NPs dose at $210 \mathrm{~min}$ contact time with $\mathrm{pH} 6$ reveals a higher percent As(III) removal which reduced with a rise in concentration of As(III). When $0.05 \mathrm{~g} \mathrm{~L}^{-1}$ mHAP NPs dose applied, 70\% removal of As(III) was achieved that further improved to $100 \%$ after employing $0.12 \mathrm{~g} \mathrm{~L}^{-1} \mathrm{mHAP}$ NPs dose. Consequently, as the mHAP NPs dose increases, more adhesive sites are available to bind the adsorbate and in the process removal percentage along with rate of adsorption also showed a upwardly trend. Fig. 6(G) showed cubic contour plot of cumulative effect of all variable parameters on percentage removal of As(III).

Analysis of variance (ANOVA) was used to determine the impact of autonomous variables on dependent variable in a regression analysis. The quadratic polynomial equation showed significant $R^{2} 0.8020$ and model $F$ value 4.34 for As(III) 
Table 3 ANOVA for response surface quadratic model for removal of As(III) from water by using $6 \mathrm{~h}$ aged mHAP NPs

\begin{tabular}{|c|c|c|c|c|c|c|}
\hline Source & Sum of mean squares & $D_{\mathrm{f}}$ & Mean square & $F$ & $p$-Value & Remarks \\
\hline Model & 8005.28 & 14 & 571.81 & 4.34 & 0.0039 & Significant \\
\hline Contact time (A) & 975.37 & 1 & 975.37 & 7.40 & 0.0158 & Significant \\
\hline $\mathrm{pH}(\mathrm{B})$ & 70.04 & 1 & 70.04 & 0.53 & 0.4771 & Insignificant \\
\hline $6 \mathrm{~h}$ aged mHAP NPs dose (C) & 2147.04 & 1 & 2147.04 & 16.30 & 0.0011 & Significant \\
\hline As(III) conc. (D) & 852.04 & 1 & 852.04 & 6.47 & 0.0225 & Significant \\
\hline $\mathrm{AB}$ & 3.06 & 1 & 3.06 & 0.023 & 0.8808 & Insignificant \\
\hline $\mathrm{AC}$ & 10.56 & 1 & 10.56 & 0.080 & 0.7809 & Insignificant \\
\hline $\mathrm{AD}$ & 18.06 & 1 & 18.06 & 0.14 & 0.7163 & Insignificant \\
\hline BC & 33.06 & 1 & 33.06 & 0.25 & 0.6236 & Insignificant \\
\hline $\mathrm{BD}$ & 14.06 & 1 & 14.06 & 0.11 & 0.7484 & Insignificant \\
\hline $\mathrm{CD}$ & 297.56 & 1 & 297.56 & 2.26 & 0.1536 & Insignificant \\
\hline $\mathrm{A}^{2}$ & 1723.57 & 1 & 1723.57 & 13.08 & 0.0025 & Significant \\
\hline $\mathrm{B}^{2}$ & 322.15 & 1 & 322.15 & 2.45 & 0.1387 & Insignificant \\
\hline $\mathrm{C}^{2}$ & 1616.57 & 1 & 1616.57 & 12.27 & 0.0032 & Significant \\
\hline $\mathrm{D}^{2}$ & 1222.86 & 1 & 1222.86 & 9.28 & 0.0082 & Significant \\
\hline Residual & 1975.92 & 15 & 131.73 & - & - & 一 \\
\hline Lack of fit & 1975.08 & 10 & 197.51 & 1185.05 & $<0.0001$ & - \\
\hline Pure error & 0.83 & 5 & 0.17 & - & - & - \\
\hline Cor total & 9981.20 & 29 & - & - & - & - \\
\hline
\end{tabular}

removal using $6 \mathrm{~h}$ mHAP NPs. The associated $p$ value is used to estimate whether $F$ is large enough to indicate statistical significance. The lack of fit is also significant due to prob $>F$ less than 0.0500. In this case $\mathrm{A}, \mathrm{C}, \mathrm{D}, \mathrm{A}^{2}, \mathrm{C}^{2}, \mathrm{D}^{2}$ are significant model terms. ANOVA for the predicted response surface quadratic model was presented in Table 3. Along with the ANOVA analysis for lack of fit test, model summary statistics also provides information regarding fairness of selected model in order to establish a relation between response and variable. In our case the significant lack of fit (1185.05) was observed which might be due to presence noise.

The "Adeq Precision" measured from the signal to noise ratio should be greater than 4 . In our case, the observed "Adeq Precision" ratio was 6.602 indicated in Table 4. Hence, the chosen model is suitable and appropriate to navigate the design space.

\subsection{Adsorption isotherm}

Adsorption isotherm is an empirical relationship which determines the distribution of adsorbate concentration between solidliquid phases. ${ }^{29}$ Isotherms also defined the relationship between the amount adsorbed by a unit weight of adsorbent and the amount of adsorbate remaining in a medium at equilibrium, at constant temperature. The batch experiments data were employed according to Langmuir, ${ }^{30}$ Freundlich ${ }^{31}$ and Temkin ${ }^{32}$ isotherms to establish adsorption hypothesis. Additional details were given in ESI Section. $\dagger$ The obtained isotherm parameters for above all theories mentioned in following Table 5.
Through the comparison between $R^{2}$ values it could be concluded that adsorption process of $6 \mathrm{~h}$ mHAP NPs is best described by Langmuir isotherm. Observed $b$ value $81.042 \mathrm{~L}$ $\mathrm{mg}^{-1}$ indicated strong interaction between As(III) ions and optimized magnetized $6 \mathrm{~h}$ mHAP NPs. Maximum binding capacity $\left(q_{\mathrm{m}}\right)$ of $6 \mathrm{~h}$ mHAP NPs was found $12.345 \mathrm{mg} \mathrm{g}^{-1}$. A comparison of the percentage removal of As(III) by using various NPs reported in the literature with present $6 \mathrm{~h}$ aged mHAP NPs is listed in Table 6 in order to highlight the superior adsorption removal of our nano system.

\subsection{Removal kinetics}

Lagergren pseudo-first order and pseudo-second order kinetics has been applied for removal of As(III) using $6 \mathrm{~h}$ mHAP NPs. The Lagergren rate equation is indicated adsorbate uptake with respect to time from a solid-liquid interface. More details could be found in ESI Section S1.3. $\dagger$ The Lagergren's first order rate constant $\left(k_{1}\right)$ and $q_{\mathrm{e}}$ are calculated from the intercept and slope of the plot and listed in Table 7 along with corresponding coefficients.

3.5.1. Intra-particle diffusion model. The intra-particle diffusion (IPD) model proposed by Weber and Morris (1962) has been applied for adsorption kinetics. ${ }^{38}$ The As metal ions are transported from the bulk of the solution into the solid phase through an intra-particle diffusion process. ${ }^{39}$

From Table 7, it can be observed that kinetic constants $\left(k_{1}\right.$, $\left.k_{2}\right)$ and $q_{\mathrm{e}}$ calculated $\left(q_{\mathrm{e}, \mathrm{cal}}\right)$ vary with $q_{\mathrm{e}}$ experimental $\left(q_{\mathrm{e}, \exp }\right)$ at equilibrium. $q_{\mathrm{e}, \exp }$ and $q_{\mathrm{e}, \mathrm{cal}}$ values should always follow similar

Table 4 Model fit summary for quadratic model statistics

\begin{tabular}{lllllllll}
\hline Metal & Std. dev. & Mean & C.V,\% & PRESS & $R^{2}$ & Adj. $R^{2}$ & Pred. $R^{2}$ & Adeq. precision \\
\hline As(III) & 11.48 & 76.60 & 14.98 & 11377.68 & 0.8020 & 0.6173 & -0.1399 & 6.602
\end{tabular}


Table 5 Isotherms parameters for adsorption of As(III) dye onto $6 \mathrm{~h}$ mHAP NPs

\begin{tabular}{|c|c|c|c|c|c|c|c|c|}
\hline \multicolumn{3}{|l|}{ Langmuir } & \multicolumn{3}{|c|}{ Freundlich } & \multicolumn{3}{|l|}{ Temkin } \\
\hline$q_{\mathrm{m}}, \mathrm{mg} \mathrm{g}^{-1}$ & $b, \mathrm{~L} \mathrm{mg}^{-1}$ & $R^{2}$ & $K_{\mathrm{f}}, \mathrm{L} \mathrm{g}^{-1}$ & $n$ & $R^{2}$ & $K_{\mathrm{t}}, \mathrm{L} \mathrm{mg}^{-1}$ & $\begin{array}{l}B_{\mathrm{t}} \\
\mathrm{kJ} \text { per mole }\end{array}$ & $R^{2}$ \\
\hline 12.345 & 81.042 & 0.993 & 290.905 & 0.211 & 0.802 & 10.499 & 1.842 & 0.748 \\
\hline
\end{tabular}

Table 6 Comparison of the percentage removal of various NPs towards the removal of As(III) $33-37$

\begin{tabular}{|c|c|c|c|}
\hline Adsorbent & Adsorption condition & Adsorption removal (\%) & References \\
\hline $\mathrm{NiFe}_{2} \mathrm{O}_{4}$ nanoparticles & $\begin{array}{l}30 \mathrm{~min}, \mathrm{pH} 9 \text {, agitation speed } 300 \mathrm{rpm} \text {. } \\
\text { As(III) concentration } 25 \mathrm{mg} \mathrm{L}^{-1} \\
\text { adsorbent dosage } 6 \mathrm{~g} \mathrm{~L}^{-1}\end{array}$ & 91 & K. Karakaş et al. $(2017)^{33}$ \\
\hline Hydrous zirconium oxide & $\begin{array}{l}10 \mathrm{~min}, \mathrm{As}(\mathrm{III}) \text { concentration } 0.105 \mathrm{mg} \mathrm{L}^{-1} \text {, } \\
\text { adsorbent dosage } 0.15 \mathrm{~g} \mathrm{~L}^{-1}\end{array}$ & 66 & Hang C. et al. $(2011)^{34}$ \\
\hline $\mathrm{Al}_{2} \mathrm{O}_{3}$ nanoparticles & $\begin{array}{l}15 \mathrm{~h}, \mathrm{As}(\mathrm{III}) \text { concentration } 5 \mathrm{mg} \mathrm{L}^{-1} \\
\text { adsorbent concentration } 2 \mathrm{~g} \mathrm{~L}^{-1}\end{array}$ & $51 \pm 5$ & Önnby L. et al. $(2014)^{35}$ \\
\hline Succinic acid engineered magnetic NPs & $\begin{array}{l}24 \mathrm{~h}, \mathrm{As}(\mathrm{III}) \text { concentration } 19.6 \mathrm{mg} \mathrm{L}^{-1} \\
\text { adsorbent dose } 1.250 \mathrm{~g} \mathrm{~L}^{-1}, \mathrm{pH} 8\end{array}$ & 91 & Singh et al. $(2011)^{37}$ \\
\hline $\begin{array}{l}\text { Hydroxyapatite capped Mn doped } \\
\text { magnetite NPs ( } 6 \text { h mHAP NPs) }\end{array}$ & $\begin{array}{l}3.5 \mathrm{~h}, \mathrm{As}(\mathrm{III}) \text { concentration } 0.5 \mathrm{mg} \mathrm{L}^{-1} \text {, } \\
\text { adsorbent dose } 0.2 \mathrm{~g} \mathrm{~L}^{-1}, \mathrm{pH} 6.5\end{array}$ & 98 & Present study \\
\hline
\end{tabular}

trends, i.e., increase with increasing concentration of initial metal ion concentration. In case of pseudo-first order kinetic model over the full range of initial metal ion concentrations, $q_{\mathrm{e}, \mathrm{cal}}$ values are not following similar trends as $q_{\mathrm{e}, \exp }$ which emphasized pseudo-first order kinetic model is not suitable for present study. In case of pseudo-second order kinetic model, $q_{\text {e,cal }}$ and $q_{\text {e,exp }}$ values showed synchronization with each other and manifested their applicability over pseudo first order kinetics.

The uptake rate of solute mainly depends on the characteristics features of adsorbent such as size, shape, surface area, charge, porosity and functional groups on the surface. According to Hameed (2009), $q_{t}$ vs. $t^{1 / 2}$ plot linearity decides the mechanism of diffusion i.e., when it passes through origin then single operating factor responsible otherwise combined factors are involved along with intra particle diffusion. ${ }^{40}$ The different stages of rate of sorption are indicative of the fact that, adsorption rate was initially faster which slowed down over a period. The plots (not shown) were not linear over the whole time range, implying that more than one process affected the adsorption mechanism. The adsorption data as showed in Table 7 indicated that removal of As(III) from aqueous phase is a complex process, involving both boundary layer diffusion as well as intra-particle diffusion.

\subsection{Thermodynamic of As(III) adsorption}

The evaluation of thermodynamics parameters, namely, enthalpy $\left(\Delta H^{\circ}\right)$, Gibbs free $\left(\Delta G^{\circ}\right)$ and entropy $\left(\Delta S^{\circ}\right)$ are used to predict practical feasibility and nature of adsorption process.

The negative values of $\Delta G^{\circ}$ indicated that As(III) adsorption onto $6 \mathrm{~h}$ mHAP NPs is following spontaneous process as

Table 7 Pseudo-first order, second order kinetic and intra-particle diffusion model parameters for adsorption of As(III) onto $6 \mathrm{~h}$ mHAP NPs

\begin{tabular}{|c|c|c|c|c|c|c|c|c|c|c|}
\hline \multirow{2}{*}{$\begin{array}{l}\operatorname{As}(\mathrm{III}), \\
\left(\mathrm{mg} \mathrm{L}^{-1}\right)\end{array}$} & \multicolumn{4}{|c|}{ Pseudo-first order kinetics } & \multicolumn{3}{|c|}{ Pseudo-second order kinetic } & \multicolumn{3}{|c|}{ Intra-particle diffusion model } \\
\hline & $q_{\mathrm{e}, \exp }$ & $q_{\mathrm{e}, \mathrm{cal}}$ & $k_{1}$ & $R^{2}$ & $q_{\mathrm{e}, \mathrm{cal}}$ & $k_{2}$ & $R^{2}$ & $C_{\mathrm{i}}$ & $K_{\mathrm{ip}}$ & $R^{2}$ \\
\hline 0.2 & 1.975 & 0.8011 & 0.020727 & 0.998 & 2.123142 & 0.02505 & 0.997 & 0.765 & 0.083 & 0.898 \\
\hline 0.3 & 2.950 & 0.9187 & 0.018424 & 0.982 & 3.184713 & 0.01540 & 0.997 & 1.080 & 0.128 & 0.905 \\
\hline 0.5 & 4.899 & 0.4323 & 0.018424 & 0.983 & 5.319149 & 0.00868 & 0.997 & 1.708 & 0.218 & 0.911 \\
\hline 0.7 & 6.642 & 0.2903 & 0.016121 & 0.994 & 7.299270 & 0.00563 & 0.996 & 2.142 & 0.414 & 0.887 \\
\hline 1.0 & 8.610 & 0.0823 & 0.018424 & 0.969 & 9.708738 & 0.00350 & 0.993 & 2.987 & 0.641 & 0.879 \\
\hline 1.5 & 10.640 & 0.0060 & 0.018424 & 0.963 & 11.76471 & 0.00355 & 0.995 & 3.457 & 0.865 & 0.912 \\
\hline 2.0 & 11.750 & 0.0335 & 0.018424 & 0.981 & 12.98701 & 0.00375 & 0.997 & 4.142 & 0.953 & 0.945 \\
\hline 2.5 & 12.672 & 0.0168 & 0.018424 & 0.96 & 13.69863 & 0.00397 & 0.998 & 4.985 & 1.201 & 0.873 \\
\hline 3.0 & 13.673 & 0.0039 & 0.013818 & 0.994 & 15.62501 & 0.00222 & 0.991 & 3.987 & 1.468 & 0.893 \\
\hline 3.5 & 14.670 & 0.1106 & 0.013818 & 0.961 & 17.54386 & 0.00146 & 0.984 & 5.980 & 1.602 & 0.899 \\
\hline 4.0 & 15.675 & 0.1881 & 0.013818 & 0.922 & 19.23077 & 0.00099 & 0.971 & 6.873 & 1.639 & 0.932 \\
\hline
\end{tabular}


a result, no extra energy required for penetration of $\mathrm{As}(\mathrm{III})$ ions into the adsorbent. The negative value of $\Delta H^{\circ}-83.2231 \mathrm{~kJ}$ $\mathrm{mol}^{-1}$ and $\Delta S^{\circ}-210.1781 \mathrm{~J} \mathrm{~mol}^{-1} \mathrm{~K}^{-1}$ established that adsorption process is exothermic in nature. As reported the change in free energy for non covalent physio-sorption is between -20 and $0 \mathrm{~kJ} \mathrm{~mol}{ }^{-1} .^{41}$ In present study $\Delta G^{\circ}$ are $-21.1677 \mathrm{~kJ} \mathrm{~mol}^{-1}$ for As(III) ions which favors ionic interaction with 6 h mHAP NPs.

\subsection{Desorption study}

$10 \mathrm{~mL} \mathrm{As(III)} \mathrm{solution} \mathrm{of} 0.5 \mathrm{mg} \mathrm{L}^{-1}$ concentration was equilibrated for $210 \mathrm{~min}$ with $0.20 \mathrm{~g} \mathrm{~L}^{-1}$ of $6 \mathrm{~h}$ mHAP NPs, after which the mHAP NPs were collected and dried under lyophilization in order to determine adsorbed As(III) amount. In desorption tests, As(III) saturated mHAP NPs were added to $10 \mathrm{~mL}$ of $0.1 \mathrm{M} \mathrm{NaOH}$, $0.1 \mathrm{M} \mathrm{H}_{2} \mathrm{SO}_{4}$ and 0.1 M EDTA regeneration solutions individually which were then left on a rotary shaker for $6 \mathrm{~h}$. Subsequently, the NPs were pulled down by applying magnetic field and supernatant was analyzed through AFS in order to find out desorbed As(III). The above procedure was repeated in order to completely chelate all the As(III) ions. The result obtained suggests EDTA as an excellent desorption tool with maximum desorption capacity around 58\% in first cycle which further increased to $99 \%$ in the subsequent cycle owing to it metal chelating property. However, alkaline and acidic conditions offered moderate desorption capacity. Alkaline condition has an edge over acidic condition because of the disturbed sorption boundary promotes leaching out the As(III) from mHAP NPs at a accelerated pace.

\section{Conclusion}

The combination of analytical chemistry and nanotechnology offers alternate viable techniques towards remediation of water. Here, highly stable, biocompatible nanotized one pot hydroxyapatite capped Mn doped SPIONs adsorbent were synthesized successfully for expeditious removal of toxic As(III) from water. Systematic batch experiments were formed following CCD response surface design to optimize the autonomous parameters in removal process. RSM model statistics summary confirmed that, developed model is acceptable well with experimental data. The optimum conditions were found to be magnetite $6 \mathrm{~h}$ aged mHAP NPs dose of $0.2 \mathrm{~g} \mathrm{~L}^{-1}$, contact time of $210 \mathrm{~min}$ and $\mathrm{As}$ (III) concentration of $0.5 \mathrm{mg} \mathrm{L}^{-1}$ in acidic media. The process followed spontaneous, monolayer saturated adsorption in pseudo-second order kinetic manner. Finally, a comprehensive comparison of similar technologies was carried out to gauge the superiority of the nanosystems. The high colloidal and thermal stability along with ease of separation could be pragmatic alternative for water decontamination and in various other industrial sectors where As(III) contamination is a concern.

\section{Acknowledgements}

The work was financially supported by the $12^{\text {th }}$ FY CSIR NETWORK project, NanoSHE (BSC0112) and Clean Water:
Sustainable Option (ESC-306). Author ND is thankful to CSIR for her fellowship.

\section{References}

1 P. Sylvester, P. Westerhoff, T. Möller, M. Badruzzaman and O. Boyd, Environ. Eng. Sci., 2007, 24, 104-112.

2 H. J. Shipley, S. Yean, A. T. Kan and M. B. Tomson, Environ. Toxicol. Chem., 2009, 28, 509-515.

3 D. Mohan and C. U. Pittman, J. Hazard. Mater., 2007, 142, 153.

4 Z. Wen, C. Dai, Y. Zhu and Y. Zhang, RSC Adv., 2015, 5, 40584068.

5 IARC Monographs on the Evaluation of Carcinogenic Risks to Humans, IARC, Lyon, 2004, pp. 1-1452.

6 S. W. Al Rmalli, C. F. Harrington, M. Ayub and P. I. Haris, J. Environ. Monit., 2005, 7, 279-282.

7 A. M. Ehrlich, Risk assessment guidelines update, NTIS, SPRINGFIELD, VA(USA)., 1988.

8 M. Zaw and M. T. Emett, Toxicol. Lett., 2002, 133, 113-118.

9 J. G. Hering, P.-Y. Chen, J. A. Wilkie and M. Elimelech, J. Environ. Eng., 1997, 123, 800-807.

10 R. Sahraei, K. Hemmati and M. Ghaemy, RSC Adv., 2016, 6, 72487-72499.

11 B. Daus, R. Wennrich and H. Weiss, Water Res., 2004, 38, 2948-2954.

12 A.-F. Ngomsik, A. s. Bee, M. Draye, G. r. Cote and V. r. Cabuil, C. R. Chim., 2005, 8, 963-970.

13 L. Cumbal and A. K. SenGupta, Environ. Sci. Technol., 2005, 39, 6508-6515.

14 T. Pradeep, Thin Solid Films, 2009, 517, 6441-6478.

15 S. C. N. Tang and I. M. C. Lo, Water Res., 2013, 47, 2613-2632.

16 J. Hu, G. Chen and I. M. C. Lo, Water Res., 2005, 39, 45284536.

17 O. Hakami, Y. Zhang and C. J. Banks, Water Res., 2012, 46, 3913-3922.

18 X. Zhang, H. Niu, Y. Pan, Y. Shi and Y. Cai, Anal. Chem., 2010, 82, 2363-2371.

19 J.-f. Liu, Z.-s. Zhao and G.-b. Jiang, Environ. Sci. Technol., 2008, 42, 6949-6954.

20 M. Islam, P. C. Mishra and R. Patel, J. Hazard. Mater., 2011, 189, 755-763.

21 M. Czerniczyniec, S. Farías, J. Magallanes and D. Cicerone, Water, Air, Soil Pollut., 2007, 180, 75-82.

22 C. Shan and M. Tong, Water Res., 2013, 47, 3411-3421.

23 A. American Public Health, A. American Water Works, F. Water Pollution Control and F. Water Environment, Standard methods for the examination of water and wastewater, American Public Health Association., 1915.

24 X. Wang and L. Andrews, J. Phys. Chem. A, 2006, 110, 1003510045.

25 S. Wang, B. Wang, H. Si, J. Shan and X. Yang, RSC Adv., 2015, 5, 8028-8036.

26 J. Reichert and J. G. P. Binner, J. Mater. Sci., 1996, 31, 12311241.

27 S. Goldberg and C. T. Johnston, J. Colloid Interface Sci., 2001, 234, 204-216. 
28 P. Das, P. Banerjee and S. Mondal, Environ. Sci. Pollut. Res., 2016, 22, 1318-1328.

29 A. Asfaram, M. Ghaedi and G. R. Ghezelbash, $R S C A d v ., 1916$, 6, 23599-23610.

30 I. Langmuir, J. Am. Chem. Soc., 1916, 38, 2221-2295.

31 H. M. F. Freundlich, J. Phys. Chem., 1906, 57, 1100-1107.

32 M. I. Temkin and V. Pyzhev, Acta Physicochim. URSS, 1940, 12, 217-222.

33 K. Karakaş, Z. Boncukcuoğlu, R. Karakaş, and I. Hakkı, Sep. Sci. Technol., 2017, 52, 21-34.

34 C. Hang, Q. Li, S. Gao and J. K. Shang, Ind. Eng. Chem. Res., 2012, 51, 353-361.

35 L. Önnby, P. S. Kumar, K. G. V. Sigfridsson, O. F. Wendt, S. Carlson and H. Kirsebom, Chemosphere, 2014, 113, 151157.
36 U. K. Sahu, M. K. Sahu, S. S. Mahapatra and R. K. Patel, Water, Air, Soil Pollut., 2016, 228, 45.

37 S. Singh, K. C. Barick and D. Bahadur, J. Hazard. Mater., 2011, 192, 1539-1547.

$38 \mathrm{~W}$. J. Weber and J. C. Morris, Proceedings of the First International Conference on Water Pollution Research, 1962.

39 M. Dö̈̈̈an, H. Abak and M. Alkan, J. Hazard. Mater., 2009, 164, 172-181.

40 B. H. Hameed and A. A. Ahmad, J. Hazard. Mater., 2009, 164, 870-875.

41 C. A. Hunter and J. K. M. Sanders, J. Am. Chem. Soc., 1990, 112, 5525-5534. 